\begin{tabular}{|c|c|}
\hline I & $\begin{array}{c}\text { International Journal of Current Research } \\
\text { and Academic Review }\end{array}$ \\
\hline $\begin{array}{l}\text { UCELIS } \\
\text { UBLLI }\end{array}$ & $\begin{array}{c}\text { ISSN: 2347-3215 (Online) Volume } 7 \text { Number } 2 \text { (February-2019) } \\
\text { Journal homepage: http://www.ijcrar.com }\end{array}$ \\
\hline
\end{tabular}

doi: https://doi.org/10.20546/ijcrar.2019.702.003

\title{
Survey of Energy-Aware Routing Model
}

\author{
Firas Almukhtar $^{1 *}$, Shadan Baban ${ }^{2}$ and Omer Mageb Mohammadhasan ${ }^{3}$ \\ ${ }^{1}$ Computer Technical Engineering, Imam Ja'afar Al-Sadiq University, Kirkuk - Iraq \\ ${ }^{2}$ Information System Engineering, Erbil Polytechnic University \\ ${ }^{3}$ North Gas Company, Ministry of Oil/Iraq \\ *Corresponding author
}

\section{Abstract}

Energy has become an important issue in many areas because of rising energy costs and environmental issues. The Internet is one of the major energy consuming areas. Energy savings are achieved by putting into sleep mode to minimize the total energy consumption of the network. In this paper we present three approaches: SDN-Based approach for energy-aware, dynamic topology for MPLS and heuristic-based optimization. And then present a comparison between these algorithms.
\end{abstract}

\section{Article Info}

Accepted: 22 January 2019

Available Online: 20 February 2019

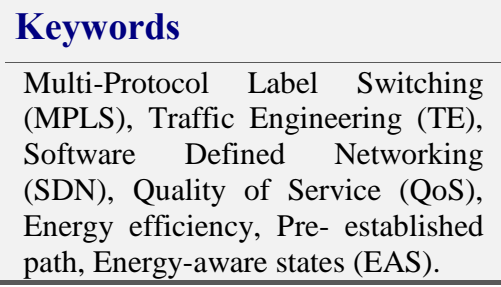

\section{Introduction}

Network operators have to allocate rapid expansion amount of their operating budget to electricity bills nowadays, and this is due to the operation of a larger number of network devices in order to meet higher traffic demands and also the increasing price of electricity (1). Operation in European telecom Currently consume 21.4 TWh per year and this is predicted to increase to $35.8 \mathrm{TWh}$ by 2020 if no green networking technologies are introduced (2).

Networks have an important role in today's highly connected world, and their traffic is expected to increase by a factor of three in the next five years. The Internet depended of many elements that are used for secure network from failures, data bursts, packet drops and delays. Some of these elements are seldom or not used at all under several traffic loads (3). Although all the nodes are active all the time, in studies shown that the capacity of links used at most $40 \%$, depending of the studies that concentrate on saving power in the Internet the proportion of Internet power exhaustion compute for up to $10 \%$ of the worldwide power exhaustion. To support the dependence between load and consumption, a strategy able to preferable achievement the network infrastructure, ensuring the QoS required without losing power, should be devised. A favorable strategy is power saving Traffic Engineering (TE) that will be carried out assuming that devices can be put to sleep. Multi-Protocol Label Switching (MPLS) is, together with the Open Shortest Path First (OSPF) protocol, the most common protocol adopted in the backbone networks. MPLS warranty a very flexible TE because it authorized to explicitly pick the route of each individual traffic demand (6).

The rest of the paper is organized as follows. In Section II present of an energy aware routing and resource management model for large-scale networks by adapting an SDN (Software 
Defined Networking) based approach. In Section III review of seeks to improve Performance Investigation of Dynamic Topologies in MPLS Networks. In Section IV describe centralize and hierarchical control frameworks for reducing power consumption in backbone computer networks. Finally, concluding remarks are exposed in Section V.

\section{SDN-based approach for energy-aware}

In this section, we present the proposed an SDN-based approach for energy-aware routing and resource management for largescale MPLS networks. In this model, ingress (IRs) and egress routers (ERs) are linked with each other by PLSPs. Core routers between IRs and ERs only forward data packets depended on labels. Controller stores PLSP information in PLSP table. Controller performs energy aware routing based on several cases that shown below:-

1. Load of a pair can be hold using least number of paths. They improve a PLSP Selection Algorithm (PSA) using dynamic programming.

2. Raise of power consumption can be delayed or completely bypass by execute load balancing across active PLSPs of an IEP.

3. Overall capacity of active paths may not be suitable to carry current traffic load. The load of an IEP may override certain threshold such as $90 \%$. This means that all paths of a pair are highly loaded. Subsequently, controller resizes all or several of active PLSPs so as to get extra space for highly loaded paths. Controller could switch on a new PLSP instead of resizing active ones so as to increase IEP capacity. However, energy consumption would increase.

Since this method is power hungry, controller tries to postpone path activation (3).

\begin{tabular}{l}
\hline Algorithm 1 PLSP Selection Algorithm \\
\hline Require: $\mathrm{Vi}(\mathrm{N}, \mathrm{C})$ denotes the optimal solution in terms \\
of energy for $\mathrm{N}$ paths with capacity $\mathrm{C}$ and load $\mathrm{Li}$ for an \\
IEPi. \\
Step 1:- Select optimal solution in terms of energy for $\mathrm{N}$ \\
paths with capacity $\mathrm{C}$ for an IEP as $\mathrm{V}(\mathrm{N}, \mathrm{C})$ \\
Step 2:- If path $\mathrm{i}_{\mathrm{i}}$ stays active then set path $=\mathrm{V}_{\mathrm{i}}(\mathrm{N}-1$, \\
C), else the path is off, set $\mathrm{C}_{\text {new }}=\mathrm{C}-\mathrm{c}_{\mathrm{i}}$ and $\mathrm{V}_{\text {new }}=\mathrm{V}_{\mathrm{i}}-\mathrm{e}_{\mathrm{i}}$. \\
Step 3:- pick $\mathrm{V}(\mathrm{N}, \mathrm{C})$ becomes min $\left(\mathrm{V}_{\mathrm{i}}(\mathrm{N}-1, \mathrm{C}), \mathrm{Vi}\right.$ \\
$\left.\left(\mathrm{N}-1, \mathrm{C}-\mathrm{c}_{\mathrm{i}}\right)-\mathrm{e}_{\mathrm{i}}\right)$. \\
Step 4:- If total capacity of selected path (i) are less than \\
Li, Then, optimum path combination in the table will be \\
$\mathrm{V}(\mathrm{N}, \mathrm{C})$.
\end{tabular}

\section{Dyamic Topologies in MPLS Network (DTM)}

In this section, we present components and functions of DTM will be introduced. The implementation relies on MPLS in combination with flow-aware label assignment (4). Only the ingress router needs to be modified to support DTM. Dynamic Topologies using MPLS consist of three components:

1. Network Management Function (NMF)

It determines reduced topologies and optimal paths. NMF execute online and measures the network utilization. The active Topology ID is broadcasted to all nodes to indicative the modification in topology the TFT and PMF functions. Once the nodes receive the messages, they perform relevant switch-over functions and start connected timers. The other components are centrally controlled by this function.

2. Topology and Flow Tracker (TFT)

The TFT is the master change in the forwarding path. It maintains flow states for all labels that have been assigned. Two timers are used by the scheme to schedule job of individual modules. The first timer distinguish how long each state is maintained for present flows when the topology is reduce in size; the second timer determines the time period until the state is erased in the flow tracker once the topology is expand.

3. Power Management Function (PMF)

This function (PMF) is work when the topologies have changed. Once the topology is downsized, the PMF function will stay the traffic flows to migrate to the new topology. After that, the node and links of that topology will be turn into a standby mode.

\section{DTM algorithm}

For the DTM algorithm, the network functions are controlled by the NMF. In case of receiving packets, the NMF will realize the Topology ID which distinguishes the network from another. If the Topology ID is the same than previous one, the NMF will apply the same label-set. In case of Topology ID is different, the NMF recognizes the new network topology that specified by the topology ID. If the number of active nodes and links in the network topology is reduced, the change is identified as networks downsize and the NMF will apply the appropriate label set. The network will continue to use the previous label-set for established flows for time $\mathrm{T}$, controlled by the TFT via the NMF. After the timer expires, the NMF will power downs the selected nodes and links in the new topology into the standby mode by using PMF function. Once the network topology status is increasing the number of active nodes and links, the NMF will select the new Topology ID and power up the network elements by controlling the PMF. The new label set will activate after the power up timer expired which is controlling by the TFT. The figure 1 has shown the flowchart of DTM. 


\section{IV- Heuristic-based optimization algorithm}

In this section we present the implementation of control framework for power saving. The decisions about activity and energy status of all devices are determined by solving the problem of decrease the power exhaustion in the whole network. The optimal network execution is computed based on known network topology and expected requested (traffic matrix). The energy optimization problem is formulated as a mathematical programming problem with various constraints and control parameters. They proposed an algorithm to solve Link-Node Heuristic Problem (LNHP). The proposed method works in two parts. First, the initial solution is determined by widely used linear solver. Second, the main problem is modified and calculations are recurrent for this modified formulation. Thus, the optimization problem LNHP is repetitively modified and solved until all decision variables take binary values - 0 or 1 , and the calculations are terminated (5). The original formulation of LNHP and its transformation to the continuous problem are presented below.

$\min _{x_{c_{2}} y_{\alpha k} z_{r i} u_{\mathrm{Rd}}}$

$$
\begin{aligned}
& \left\{F_{L N H}=\sum_{e=1}^{E} \sum_{k=1}^{K} \varepsilon_{e k} y_{e k}+\sum_{c=1}^{C} W_{c} x_{c}+\sum_{r=1}^{R} T_{\gamma} z_{y}\right\} \\
& \forall_{e=1} E \quad Y_{e 1} \geq Y_{e i} \geq{ }^{\cdots \times} y_{e k} \geq
\end{aligned}
$$

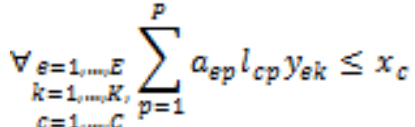

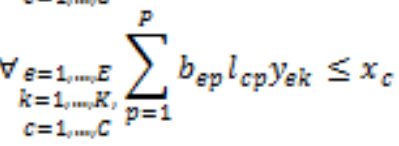

where $\mathrm{T}=1, \ldots, \mathrm{R}$ denote routers, $\mathrm{c}=1, \ldots, \mathrm{C}$ cards, $\mathrm{p}=1, ., \mathrm{P}$ ports and $\mathrm{e}=1, \ldots, \mathrm{E}$ links in a network. Each router contains $\mathrm{C}$ cards and each card contains $\mathrm{P}$ ports. Routers, cards and ports can operate in one of $\mathrm{K}$ energy states - EAS $(\mathrm{k}=1, \ldots, \mathrm{K})$. All routers and cards can work in two phases: active and sleeping $(K=2)$ and ports can work in at least two states $(K \geq 2)$. Two ports connected by the e-th link are in the same state k. $\varepsilon_{\mathrm{ek}}$ denote power consumption of link e in state $\mathrm{k}$, Wc and $\mathrm{Tr}$ fixed power levels associated to card $\mathrm{c}$ and router $\mathrm{r}$.

Fig.1 DTM flow chart

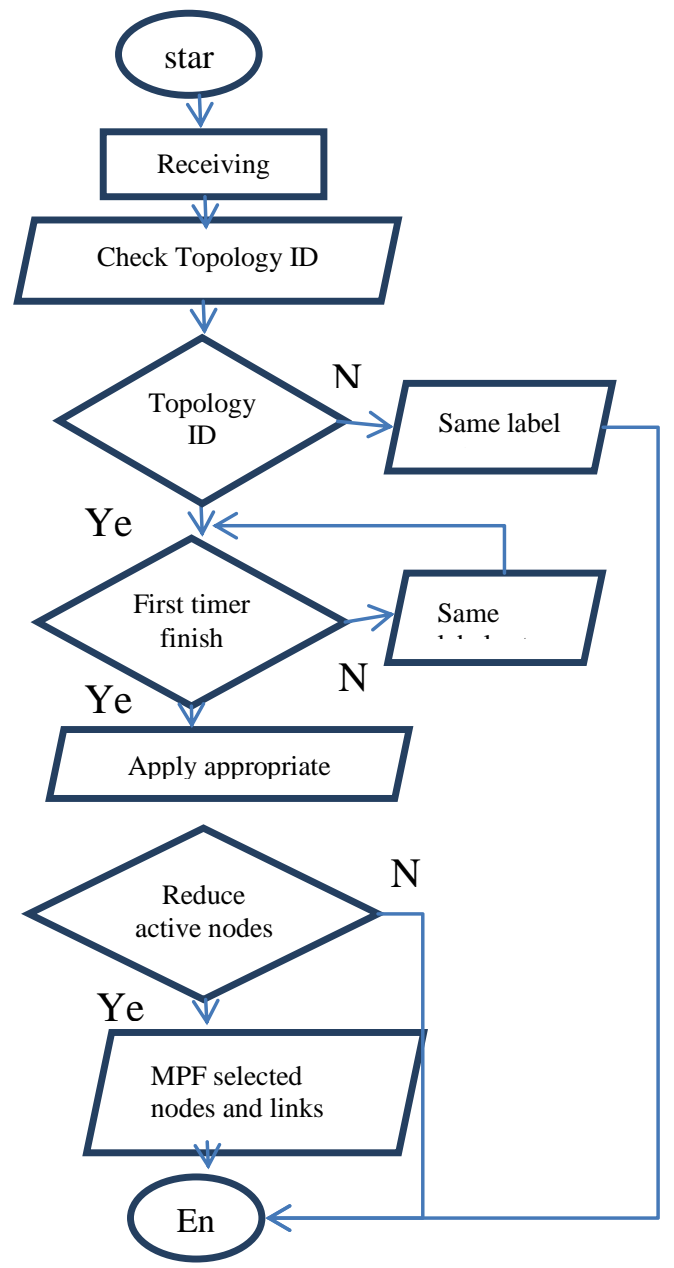


Table.1 Comparison between proposed method

\begin{tabular}{|c|c|c|c|}
\hline Name & SDN & DTM & LNHP \\
\hline Protocol & $\begin{array}{l}\text { PLSPs and } \\
\text { RSVP-TE }\end{array}$ & LSR & LNP \\
\hline $\begin{array}{c}\text { Type of } \\
\text { operation }\end{array}$ & online & $\begin{array}{c}\text { First offline } \\
\text { Second } \\
\text { online }\end{array}$ & $\begin{array}{c}\text { First offline } \\
\text { Second } \\
\text { online }\end{array}$ \\
\hline $\begin{array}{c}\text { Type of } \\
\text { developing }\end{array}$ & $\begin{array}{c}\text { routers } \\
\text { between IRs } \\
\text { and } \\
\text { ERs(develo } \\
\text { p a PLSP) }\end{array}$ & $\begin{array}{c}\text { Ingress } \\
\text { router(IR) }\end{array}$ & $\begin{array}{c}\text { central } \\
\text { decision unit }\end{array}$ \\
\hline $\begin{array}{c}\text { Type of } \\
\text { programmi } \\
\text { ng }\end{array}$ & $\begin{array}{c}\text { Dynamic } \\
\text { Programmin } \\
\mathrm{g}\end{array}$ & linear & $\begin{array}{l}\text { branch-and- } \\
\text { bound }\end{array}$ \\
\hline $\begin{array}{c}\text { Network } \\
\text { size }\end{array}$ & Small size & Small size & $\begin{array}{l}\text { Medium } \\
\text { size }\end{array}$ \\
\hline $\begin{array}{l}\text { Saving } \\
\text { power }\end{array}$ & $\begin{array}{c}\text { Based on } \\
\text { aggregated } \\
\text { traffic load }\end{array}$ & $\begin{array}{l}\text { According to } \\
\text { the length of } \\
\text { the timer. }\end{array}$ & $\begin{array}{l}\text { continuous } \\
\text { optimization }\end{array}$ \\
\hline
\end{tabular}

The Pseudo-code of LNHP is given below.

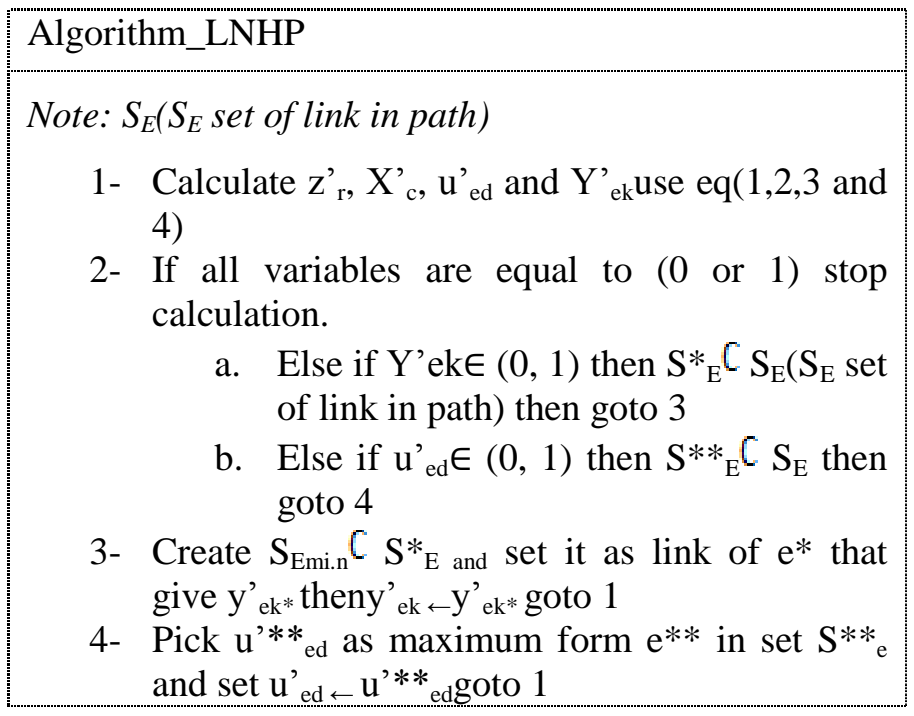

\section{V-Comparison between the algorithms}

The following shown the comparison in table 1 depended on protocol connection between each ingress and egress pair (IEPs) and types of algorithms for selecting path, types of programming amount of energy aware of the above algorithms.(3)(4)(5)

In conclusions, we present three type of proposed method for minimizing the power cost of network for MPLS utilizing. The first proposed method is an SDNbased approach for power saving routing and management of resource for largescale MPLS networks, this method used PLSPs and RSVP-TE protocol and its work starting reducing the link of path to increase power saving then used time delay for shifting the high capacity load then used resizing if the capacity of the load exceed more than threshold, the developing part is the routers between IRs and ERs (develop a PLSP), the implementation is recommended for small size network. The second proposed method is DTM algorithm, it change the topology of routing depended on the capacity of load, it start with first topology and calculate the capacity of the load if its small they change the topology to reduce the link in the path and saving the power, the development is only of the Ingress router (IR), it recommended to use for small size of network and the amount of power saving based on the length of the timer. The last proposed method is Heuristic-based Optimization Algorithm, this method starting in the offline and used formulation for calculate the parameter and the value of the parameter is binary, and used recurrent to minimize the power consumption and continue minimizing (branch-and-bound), its used for medium size network, the development part is central decision unit.

\section{References}

1. R. Bolla et al., "The potential impact of green technologies in next generation wireline networks: Is there room for energy saving optimization?" 
IEEE Com. Mag., vol. 49, no. 8, pp. 80-86, Aug. 2011.

2. C. Lange, D. Kosiankowski, R. Weidmann, and A. Gladisch, "Energy consumption of telecommunication networks and related improved options," IEEE J. Sel. Topics Quantum Electron., vol. 17, no. 2, pp. 285-295, Mar.2011.

3. M. Rasih Celenlioglu, S. Burak Goger and H. Ali Mantar "An SDN-based Energy-Aware Routing Model for Intra-Domain Networks", Software, Telecommunications and Computer Networks (SoftCOM), $201422^{\text {nd }}$ International Conference.

\section{How to cite this article:}

Firas Almukhtar, Shadan Baban and Omer Mageb Mohammadhasan. 2019. Survey of Energy-Aware Routing Model. Int.J.Curr.Res.Aca.Rev. 7(2), 16-20.

doi: https://doi.org/10.20546/ijcrar.2019.702.003
4. Abdelnour Aldraho, Alexander A. Kist and Andrew Maxwell "Performance Investigation of Dynamic Topologies in MPLS Networks", 2012 International Symposium on Communications and Information Technologies (ISCII).

5. Ewa Niewiadomska-Szynkiewicz, Andrzej Sikora, Piotr Arabas, Mariusz Kamola, Krzysztof Malinowski, Przemyslaw lask6la, Michal Marks "Network-Wide Power Management in Computer Networks", 2013 22nd ITC Specialist Seminar on Energy Efficient and Green Networking (SSEEGN). 